\begin{tabular}{|c|l|}
\hline Title & ON A BOUND FOR A MPLITUDES OF NA VIER-STOKES FLOW WITH ALMOST PERIODIC INITIAL DATA \\
\hline Author(s) & Giga, Y oshikazu; Mahal ov, A lex; Y oneda, Tsuy oshi \\
\hline Citation & Hokkaido University Preprint Series in Mathematics, 952, 1-10 \\
\hline Issue Date & 2009-12-8 \\
\hline DOI & 10.14943/84099 \\
\hline Doc URL & http://hdl.handle.net/2115/69759 \\
\hline Type & bulletin (article) \\
\hline File Information & pre952.pdf \\
\hline
\end{tabular}

Instructions for use 


\title{
ON A BOUND FOR AMPLITUDES OF NAVIER-STOKES FLOW WITH ALMOST PERIODIC INITIAL DATA
}

\author{
YOSHIKAZU GIGA, ALEX MAHALOV, AND TSUYOSHI YONEDA
}

\begin{abstract}
For any bounded (real) initial data it is known that there is a unique global solution to the two dimensional Navier-Stokes equations. This paper is concerned with a bound for the sum of the modulus of amplitudes when initial velocity is spatially almost periodic in $2 \mathrm{D}$. In the case of general dimension, it is bounded on local time of existence shown by Giga, Inui, Mahalov and Matsui in 2005. A class of initial data is given such that the sum of the modulus of amplitudes of a solution is bounded on any finite time interval. It is shown by an explicit example that such a bound may diverge to infinity as the time goes to infinity at least for complex initial data.
\end{abstract}

AMS Subject Classification(2000):76D05,35Q30,42B05

Key words: Navier-Stokes equations, almost periodic initial data, frequency sets and amplitudes

\section{INTRODUCTION}

A unique local-in-time classical solution of the Cauchy problem for the Navier-Stokes equations in any dimension is known to exist for any bounded (real) initial data (see $[9,19])$. It was shown in $[16]$ that the solution is always spatially almost periodic at any time provided that the smooth solution exists since the solution continuously depends on initial data. This persistency property of almost periodicity also holds for the threedimensional Navier-Stokes equations in a rotating frame with almost periodic initial data even if they are complex. It was shown in [15] for $n \geq 2$ that the local-in-time solution is analytic in time. When the initial data is almost periodic, this implies that the complex amplitude is analytic in time. In particular, a new mode cannot be created at any positive time. Although in 2D case the solution is bounded in space and any finite time interval, it is an open problem whether or not the sum of modulus of amplitudes is bounded in any finite time interval provided that the corresponding quantity of almost periodic (real) initial data is finite.

In this paper we study bounds of the sum of modulus of amplitudes of a global-in-time solution of the 2D-Navier-Stokes equations:

$$
\left\{\begin{array}{l}
u_{t}-\Delta u+(u, \nabla) u+\nabla \pi=0, \quad t>0, \quad x \in \mathbb{R}^{2}, \\
\operatorname{div} u=0, \quad x \in \mathbb{R}^{2}, \\
\left.u\right|_{t=0}=u_{0},
\end{array}\right.
$$

when initial data is a real, spatially almost periodic function of the form

$$
u_{0}(x)=\sum_{m \in \mathbb{Z}^{\ell}} c_{m} \exp \left(i \sum_{\substack{j=1 \\ 1}}^{\ell} m_{j}\left(r_{j} \cdot x\right)\right), \quad x \in \mathbb{R}^{2},
$$


where $\left\{c_{m}\right\}_{m \in \mathbb{Z}^{\ell}}=\left\{c_{m}^{1}, c_{m}^{2}\right\}_{m \in \mathbb{Z}^{\ell}} \subset \mathbb{C}^{2}, r_{j} \in \mathbb{R}^{2}(j=1, \cdots, \ell)$ and $\left\{r_{j}\right\}_{j=1}^{\ell}$ is linearly independent over the field of all rational number $\mathbb{Q}$. Here $u=u(t, x)=\left(u^{1}(t, x), u^{2}(t, x)\right)$ and $\pi=\pi(t, x)$ are unknown functions of the velocity of the fluid and its pressure respectively; $u_{0}=u_{0}(x)=\left(u_{0}^{1}(x), u_{0}^{2}(x)\right)$ is a given initial velocity vector field. In the case of general dimension, the sum of the modulus of amplitudes of a solution is bounded on local time of existence (see [11]).

As we mentioned in the first paragraph, it is known that the problem (1) admits a unique global-in-time smooth solution for any bounded measurable initial data ([9]); see also [23] for improvement and [24] for extension to Boussinesq flow. Uniqueness is guaranteed by imposing structure or growth of the pressure field (see $[9,22]$ ).

We are interested in obtaining bounds of the sum of the modulus of amplitudes

$$
\sum_{m \in \mathbb{Z}^{\ell}}\left|c_{m}(t)\right|
$$

for any bounded time interval $[0, T]$. Note that this quantity equals to the $F M$ norm of $u(t)$, Fourier preimage of all finite Radon measures proposed by [11]. Our goal is to find a class of real initial data such that the above quantity $\|u(t)\|_{F M}$ is bounded in $[0, T]$ for any $T>0$. For this purpose we extend an almost periodic function $u_{0}$ to a periodic function of more variables by adding parameters. This device is proposed by [8]. We impose that the initial data is in the periodic Sobolev space and prove that the solution belongs to this Sobolev space by deriving a new a priori estimate including additional parameters. Applying the Sobolev embedding theorem we get a desired bound on the sum of modulus of amplitudes. It is not clear whether this bound is uniform with respect to real initial data if one merely bounds the sum of modulus of initial amplitudes. If an initial data is allowed to be complex, we can show that the $F M$ norm of the solution may diverge to infinity as the time goes to infinity by giving an explicit example (see Theorem 3.1).

As an application we strengthen the result of [25]. In [25] the third author showed that existence on long time intervals of regular solutions to the 3D-Navier-Stokes equations in a rotating frame with spatially almost periodic data provided that the Coriolis force is large enough by assuming that there is a bounded for $\|u(t)\|_{F M}$ in $[0, T]$ for the sum of modulus of amplitudes of a solution $u(t)$, i.e., the 2D-Navier-Stokes equations. However, existence of such a bound is not given unless initial data is periodic. The 3D-Navier-Stokes equations with the Coriolis force are described as follows:

$$
\left\{\begin{array}{l}
\partial_{t} v+(v, \nabla) v+\Omega e_{3} \times v-\Delta v=-\nabla p, \\
\nabla \cdot v=0,\left.v\right|_{t=0}=v_{0}
\end{array}\right.
$$

where $v=v(x, t)=\left(v^{1}(x, t), v^{2}(x, t), v^{3}(x, t)\right)$ is the unknown velocity vector field and $p=p(x, t)$ is the unknown scalar pressure at the point $x=\left(x_{1}, x_{2}, x_{3}\right) \in \mathbb{R}^{3}$ in space and time $t>0$ while $v_{0}=v_{0}(x)$ is the given initial velocity field. Here $\Omega \in \mathbb{R}$ is the Coriolis parameter, which is twice the angular velocity of the rotation around the vertical unit vector $e_{3}=(0,0,1)$, the kinematic viscosity coefficient in normalized by one. By $\times$ we denote the exterior product, and hence, the Coriolis term is represented by $e_{3} \times u=J u$ with the corresponding skew-symmetric $3 \times 3$ matrix $J$.

We note that Babin, Mahalov and Nicolaenko [2] considered global solvability of (3) with periodic initial data (see also $[1,3,4,5,21]$ ). They proved existence on infinite intervals of regular solutions to the 3D-Navier-Stokes equations with a large (but finite) 
Coriolis parameter with no smallness assumption on the initial data. Chemin, Desjardins, Gallagher and Grenier [7] considered the initial data decaying at space infinity and proved a global existence result. The almost periodic case is very different from periodic case and the case of decaying at the space infinity.

If initial data is allowed to be complex, results related to existence of global-in-time solution is quite different since energy estimate is no longer available. In fact, Li and Sinai [20] constructed a finite time blow-up solution of (1) for $n=3$; however, their proof still works for two-dimensional problem. More precisely, let

$$
X:=\left\{u \in L^{2}: \operatorname{supp} \hat{u} \text { is compact. }\right\} .
$$

Note that $F M \subset X$. They showed that there exists $u_{0} \in X$ such that $\|u(t)\|_{L^{2}} \rightarrow \infty$ and $\int_{0}^{t}\|\nabla u(\tau)\|_{L^{2}} d \tau \rightarrow \infty$ as $t \rightarrow T$ for some $T<\infty$. However, it seems not clear whether or not $F M$-norm of their solution blows up.

\section{Function spaces and Main REsult}

In this section, we define function spaces for almost periodic functions, and state a main result. Let us define $F M$ and $F M_{0}$ spaces as follows (see also [11]).

Definition 2.1. Let

$$
\begin{gathered}
F M:=\left\{u \in \mathcal{S}^{\prime}:\|u\|_{F M}<\infty\right\} \\
\|u\|_{F M}:=\sup \left\{|\langle u, \phi\rangle|: \phi \in C_{\infty}\left(\mathbb{R}^{n}\right) \text { and }\|\phi\|_{L^{\infty}} \leq 1\right\},
\end{gathered}
$$

where $C_{\infty}$ is the space of all (complex-valued) continuous functions that converge to zero at the space infinity (equipped with the supremum norm $\|\cdot\|_{L^{\infty}}$ ). Let

$$
F M_{0}:=\left\{u \in F M:\left\langle u, \phi_{j}\right\rangle \rightarrow 0(j \rightarrow \infty)\right\},
$$

where $\left\{\phi_{j}\right\}_{j=1}^{\infty} \subset C_{\infty}\left(\mathbb{R}^{n}\right)$ with $\operatorname{supp} \phi_{j} \subset[-1 / j, 1 / j)^{n}$ and $\left\|\phi_{j}\right\|_{L^{\infty}} \leq 1$ for $j=1,2, \cdots$.

Remark 2.2. $F M_{0}$ is a closed subspace of $F M$ with the norm $\|\cdot\|_{F M}$.

Remark 2.3. If $u$ is an almost periodic function in $F M_{0}$, namely, if $u$ is expressed as $u=\sum_{\lambda \in \Lambda \backslash\{0\}} c_{\lambda} e^{i \lambda \cdot x}$ by using a countable set $\Lambda$ in $\mathbb{R}^{3}$, then

$$
\|u\|_{F M}=\sum_{\lambda \in \Lambda \backslash\{0\}}\left|c_{\lambda}\right|
$$

Definition 2.4. For $\left\{r_{j}\right\}_{j=1}^{\ell} \subset \mathbb{R}^{2}$ (linearly independent over $\mathbb{Q}$ ), let

$$
D^{\ell}:=\left\{\vec{s} \in \mathbb{R}^{2 \ell}: 0 \leq r_{j} \cdot s_{j} \leq 2 \pi \text { for all } j=1,2, \cdots, \ell\right\} .
$$

Remark 2.5. The set $D^{\ell}$ is fundamental domain of $\mathbb{T}^{\ell} \times \mathbb{R}^{\ell}=\mathbb{R}^{2 \ell} /\left(2 \pi \mathbb{Z} \mathbf{e}_{1}+\cdots 2 \pi \mathbb{Z} \mathbf{e}_{\ell}\right)$, where we set $\mathbf{e}_{j}:=\hat{r}_{j} /\left|r_{j}\right|$ and $\hat{r}_{j}:=(\underbrace{\mathbf{0}, \cdots, \mathbf{0}}_{j-1}, r_{j}, \underbrace{\mathbf{0}, \cdots, \mathbf{0}}_{\ell-j}) \in \mathbb{R}^{2 \ell}, \mathbf{0}=(0,0) \in \mathbb{R}^{2}$. Note that $\left\{\mathbf{e}_{j}\right\}_{j=1}^{\ell}$ is an orthonormal system. We choose $\left\{\mathbf{e}_{j}\right\}_{j=\ell+1}^{2 \ell}$ such that $\left\{\mathbf{e}_{j}\right\}_{j=1}^{2 \ell}$ become orthonormal basis of $\mathbb{R}^{2 \ell}$. For $\vec{s}=\left(s_{1}, \cdots, s_{\ell}, s_{\ell+1}, \cdots, s_{2 \ell}\right) \in \mathbb{R}^{2 \ell}$, we easily see that $\vec{s}$ is expressed as $\sum_{j=1}^{2 \ell} a_{j} \mathbf{e}_{j}$, where $a_{j}:=\left(\vec{s}, \mathbf{e}_{j}\right)=\hat{r}_{j} \cdot \vec{s} /\left|r_{j}\right|=r_{j} \cdot s_{j} /\left|r_{j}\right|$. For $f: \mathbb{R}^{2 \ell} \rightarrow \mathbb{C}^{2}$ we define $\bar{f}: \mathbb{R}^{2 \ell} \rightarrow \mathbb{C}^{2}$ as $\bar{f}\left(a_{1}, \cdots, a_{2 \ell}\right):=f(\vec{s})$ for $\vec{s} \in \mathbb{R}^{2 \ell}$. We say that the function $f$ is $D^{\ell}$-periodic if

$$
\bar{f}\left(a_{1}+2 \pi m_{1}, \cdots, a_{\ell}+2 \pi m_{\ell}, a_{\ell+1}, \cdots, a_{2 \ell}\right)=\bar{f}\left(a_{1}, \cdots, a_{\ell}, a_{\ell+1}, \cdots, a_{2 \ell}\right)
$$

for any $m=\left(m_{1}, \cdots, m_{\ell}\right) \in \mathbb{Z}^{\ell}$ and $\left(a_{1}, \cdots, a_{2 \ell}\right) \in \mathbb{R}^{2 \ell}$. 
Definition 2.6. Let us define the function spaces as follows,

$L_{x}^{\infty}\left(W_{\vec{s}}^{\eta, \infty}\right)\left(\mathbb{R}^{2} \times D^{\ell}\right):=\left\{f(\cdot, \cdot): \mathbb{R}^{2} \times D^{\ell} \rightarrow \mathbb{C}^{2}: f\right.$ is $D^{\ell}$-periodic, $\left.\sum_{|k| \leq \eta}\left\|\nabla_{\vec{s}}^{k} f(x, \vec{s})\right\|_{L_{x, \vec{s}}^{\infty}}<\infty\right\}$, $L_{x}^{\infty}\left(W_{\vec{s}}^{\eta, 2}\right)\left(\mathbb{R}^{2} \times D^{\ell}\right):=\left\{f(\cdot, \cdot): \mathbb{R}^{2} \times D^{\ell} \rightarrow \mathbb{C}^{2}: f\right.$ is $D^{\ell}$-periodic, $\left.\sum_{|k| \leq \eta}\left\|\nabla_{\vec{s}}^{k} f(x, \vec{s})\right\|_{L_{x}^{\infty}\left(L_{\vec{s}}^{2}\right)}<\infty\right\}$ and

$$
\begin{aligned}
X^{\eta}\left(\mathbb{R}^{2} \times D^{\ell}\right):=\left\{f \in L_{x}^{\infty}\left(W_{\vec{s}}^{\eta, \infty}\right)\left(\mathbb{R}^{2} \times D^{\ell}\right) \cap L_{x}^{\infty}\left(W_{\vec{s}}^{\eta, 2}\right)\left(\mathbb{R}^{2} \times D^{\ell}\right):\right. \\
\left.\|f\|_{X^{\eta}}:=\sum_{|k| \leq \eta}\left\|\nabla_{\vec{s}}^{k} f(x, \vec{s})\right\|_{L_{x, \vec{s}}^{\infty}}+\sum_{|k| \leq \eta}\left\|\nabla_{\vec{s}}^{k} f(x, \vec{s})\right\|_{L_{x}^{\infty}\left(L_{\vec{s}}^{2}\right)}<\infty\right\} .
\end{aligned}
$$

Remark 2.7. It is easy to see that

$$
L_{x}^{\infty}\left(W_{\vec{s}}^{\eta, \infty}\right)\left(\mathbb{R}^{2} \times D^{\ell}\right) \subset L_{x}^{\infty}\left(W_{\vec{s}}^{\eta, 2}\right)\left(\mathbb{R}^{2} \times D^{\ell}\right) .
$$

Definition 2.8. We usually denote the following equality:

$$
u^{\vec{s}}(x)=\sum_{m \in \mathbb{Z}^{\ell}} c_{m} \exp \left(i \sum_{j=1}^{\ell} m_{j}\left(r_{j} \cdot\left(x-s_{j}\right)\right)\right)
$$

if the function $u^{\vec{s}}$ satisfies the following convergence:

$$
\lim _{N \rightarrow \infty}\left\|u^{\vec{s}}(x)-\sum_{m \in \mathbb{Z}^{\ell},|m| \leq N} c_{m} \exp \left(i \sum_{j=1}^{\ell} m_{j}\left(r_{j} \cdot\left(x-s_{j}\right)\right)\right)\right\|_{L_{\vec{s}}^{2}}=0 \text { for a.e. } x \in \mathbb{R}^{2 \ell} .
$$

Remark 2.9. If there is $x$ such that $u^{\vec{s}}(x)=\sum_{m \in \mathbb{Z}^{\ell}} c_{m} \exp \left(i \sum_{j=1}^{\ell} m_{j}\left(r_{j} \cdot\left(x-s_{j}\right)\right)\right) \in$ $L_{\vec{s}}^{2}\left(D^{\ell}\right)$, then $\left\|u^{\vec{s}}(y)\right\|_{L_{\vec{s}}^{2}\left(D^{\ell}\right)}=\left\|u^{\vec{s}}\left(y^{\prime}\right)\right\|_{L_{\vec{s}}^{2}\left(D^{\ell}\right)}$ for any $y, y^{\prime} \in \mathbb{R}^{2}$.

Remark 2.10. Let $u(x)=\sum_{m \in \mathbb{Z}^{\ell}} c_{m} \exp \left(i \sum_{j=1}^{\ell} m_{j}\left(r_{j} \cdot x\right)\right)$ and $u^{\vec{s}}(x)=\sum_{m \in \mathbb{Z}^{\ell}} c_{m} \exp \left(i \sum_{j=1}^{\ell} m_{j}\left(r_{j} \cdot\left(x-s_{j}\right)\right)\right)$. Since the function $u^{\vec{s}}(0)$ is periodic in $\vec{s} \in D^{\ell}$, we can use Sobolev's embedding theorem. Then we have

$$
\begin{aligned}
& \|u(x)\|_{F M_{x}\left(\mathbb{R}^{2}\right)}=\left\|u^{\vec{s}}(0)\right\|_{F M_{\vec{s}}\left(\mathbb{R}^{2 \ell}\right)}=\sum_{m \in \mathbb{Z}^{\ell}}\left|c_{m}\right| \\
& \leq\left(\sum_{m \in \mathbb{Z}^{\ell}}\left(1+|m|^{2}\right)^{\eta}\left|c_{m}\right|^{2}\right)^{1 / 2}\left(\sum_{m \in \mathbb{Z}^{\ell}}\left(1+|m|^{2}\right)^{-\eta}\right)^{1 / 2} \leq C_{\eta} \sum_{k \leq \eta}\left\|\nabla_{\vec{s}}^{k} u^{\vec{s}}(0)\right\|_{L_{\vec{s}}^{2}\left(D^{\ell}\right)} \\
& \leq C_{\eta} \sum_{k \leq \eta}\left\|\nabla_{\vec{s}}^{k} u^{\vec{s}}(x)\right\|_{L_{x}^{\infty}\left(L_{\vec{s}}^{2}\right)} \leq C_{\eta}\left\|u^{\vec{s}}\right\|_{X \eta\left(\mathbb{R}^{2} \times D^{\ell}\right)} \quad \text { for } \quad \eta>\ell / 2 .
\end{aligned}
$$

Remark 2.11. If the frequency set $\Lambda$ of the function $u$ has only finite element, then $u^{\vec{s}} \in X^{k}\left(\mathbb{R}^{2} \times D^{\ell}\right)$ for any $k \in \mathbb{Z}_{+}$.

Now we state the main theorem. 
Theorem 2.12. Let $u_{0}^{\vec{s}}=\sum_{m \in \mathbb{Z}^{\ell}} c_{m} \exp \left(i \sum_{j=1}^{\ell} m_{j}\left(r_{j} \cdot\left(x-s_{j}\right)\right)\right) \in X^{\eta}\left(\mathbb{R}^{2} \times D^{\ell}\right)(\eta>$ $\ell / 2$ ) with $c_{0}=0$ (note that $u_{0}(x):=\left.u_{0}^{\vec{s}}(x)\right|_{\vec{s}=(\mathbf{0}, \cdots, \mathbf{0})} \in F M_{0}\left(\mathbb{R}^{2}\right)$ ). Then the unique (mild) solution $u^{\vec{s}}(t, x)$ to the equation (1) (with initial data $u_{0}^{\vec{s}}(x)$ ) is expressed as

$$
u^{\vec{s}}(t, x)=\sum_{m \in \mathbb{Z}^{\ell} \backslash\{0\}} c_{m}(t) \exp \left(i \sum_{j=1}^{\ell} m_{j}\left(r_{j} \cdot\left(x-s_{j}\right)\right)\right) .
$$

Moreover the solution $u^{\vec{s}}$ is in $L_{l o c}^{\infty}\left([0, \infty): X^{\eta}\left(\mathbb{R}^{2} \times D^{\ell}\right)\right)$ if $u_{0}^{\vec{s}}$ is real. More precisely,

$$
\sup _{0<t \leq T}\left\|u^{\vec{s}}\right\|_{X^{\eta}\left(\mathbb{R}^{2} \times D^{\ell}\right)}(t) \leq C,
$$

where $C>0$ is depending only on $T>0$ and $\left\|u_{0}^{\vec{s}}\right\|_{X^{\eta}\left(\mathbb{R}^{2} \times D^{\ell}\right)}$.

Remark 2.13. Let $u$ be the solution to the equation (1).

If $u_{0}^{\vec{s}}=\sum_{m \in \mathbb{Z}^{\ell}} c_{m} \exp \left(i \sum_{j=1}^{\ell} m_{j}\left(r_{j} \cdot\left(x-s_{j}\right)\right)\right) \in X^{\eta}\left(\mathbb{R}^{2} \times D^{\ell}\right)$ with $c_{0}=0$, we have

$$
u(\cdot, t) \in C\left([0, \infty): F M_{0}\left(\mathbb{R}^{2}\right)\right) \quad \text { for } \quad u(x, 0)=u_{0}(x)=\left.u_{0}^{\vec{s}}(x)\right|_{\vec{s}=(\mathbf{0}, \cdots, \mathbf{0})}
$$

by Remark 2.10. In particular, the sum $\sum_{m \in \mathbb{Z}^{\ell} \backslash\{0\}}\left|c_{m}(t)\right|$ is bounded in any finite time interval $[0, T]$.

Remark 2.14. We give an example. The almost periodic (not periodic) function $u_{0}=$ $\alpha \cos \left(x_{1}+x_{2}+x_{3}\right)+\alpha \cos \left(\sqrt{2}\left(x_{1}+x_{2}+x_{3}\right)\right), \alpha=(1,1,-2)$ satisfies Remark 2.11. Thus the solution $u(\cdot, t)$ to the equation $(1)$ is in $C\left([0, \infty): F M_{0}\left(\mathbb{R}^{2}\right)\right)$ by Remark 2.13.

Proof of Theorem 2.12. Let $S(t) u_{0}^{\vec{s}}=u^{\vec{s}}$ and $U(x, \vec{y}, t):=I_{\vec{s} \rightarrow \vec{y}-\rho(x)} S(t) u^{\vec{s}}(x)$, where $I_{\vec{x} \rightarrow \vec{y}} u(\vec{x})=u(\vec{y})$ and $\rho(x)=(x, x, \cdots, x)$. Now we show that the function $U(s, \vec{y}, t)$ is expressed as the following periodic function:

$$
U(x, \vec{y}, t)=U(\vec{y}, t)=\sum_{m \in \mathbb{Z}^{\ell}} c_{m}(t) \exp \left(i \sum_{j=1}^{\ell} m_{j}\left(r_{j} \cdot y_{j}\right)\right), c_{m}(0)=c_{m} .
$$

First we show that the function $U(x, \vec{y}, t)$ is periodic with respect to $\vec{y}$. By using the uniqueness result of the mild solutions (see [9, 18, 22]), we have

$$
I_{\vec{s} \rightarrow \vec{w}} S(t) u_{0}^{\vec{s}}(x)=S(t) I_{\vec{s} \rightarrow \vec{w}} u_{0}^{\vec{s}}(x) .
$$

Since $u_{0}^{\vec{s}+\vec{w}}(x)=u_{0}^{\vec{s}}(x)$ for $\vec{w}=\left(w_{1} \tilde{m}_{1}, \cdots, w_{\ell} \tilde{m}_{\ell}\right)$, where $w_{j}=2 \pi r_{j} /\left|r_{j}\right|^{2}$ and $\tilde{m}_{j} \in \mathbb{Z}$, we see that

$$
\begin{aligned}
& U(x, \vec{y}+\vec{w})=I_{\vec{s} \rightarrow \vec{y}+\vec{w}-\rho(x)} S(t) u_{0}^{\vec{s}}(x) \\
& \quad=I_{\vec{s} \rightarrow \vec{y}-\rho(x)} I_{\vec{s} \rightarrow \vec{s}+\vec{w}} S(t) u_{0}^{\vec{s}}(x)=I_{\vec{s} \rightarrow \vec{y}-\rho(x)} S(t) I_{\vec{s} \rightarrow \vec{s}+\vec{w}} u_{0}^{\vec{s}}(x)=I_{s \rightarrow y-\rho(x)} S(t) u_{0}^{\vec{s}+\vec{w}}(x) \\
& =I_{s \rightarrow y-\rho(x)} S(t) u_{0}^{\vec{s}}(x)=U(x, \vec{y}) .
\end{aligned}
$$

Next we show that $U(x, \vec{y}, t)=U(\vec{y}, t)$. By $(6)$, we also see that

$$
\begin{gathered}
U(x+c, \vec{y}, t)=I_{x \rightarrow x+c} I_{\vec{s} \rightarrow \vec{y}-\rho(x)} S(t) u_{0}^{\vec{s}}(x)=I_{\vec{s} \rightarrow \vec{y}-(\rho(x)+\rho(c))} S(t) u_{0}^{\vec{s}}(x+c) \\
=I_{\vec{s} \rightarrow \vec{y}-\rho(x)} I_{\vec{s} \rightarrow \vec{s}-\rho(c)} S(t) u_{0}^{\vec{s}}(x+c)=I_{\vec{s} \rightarrow \vec{y}-\rho(x)} S(t) I_{\vec{s} \rightarrow \vec{s}-\rho(c)} u_{0}^{\vec{s}}(x+c) \\
=I_{\vec{s} \rightarrow \vec{y}-\rho(x)} S(t) u_{0}^{\vec{s}-\rho(c)}(x+c) \text { for all } c \in \mathbb{R}^{2}, \\
5
\end{gathered}
$$


where we used $S(t) u_{0}^{\vec{s}}(x+c)=S(t) u_{0}^{\vec{s}+\rho(c)}(x)$ since the equation (1) is translation invariant. Thus,

$$
U(x+c, \vec{y}, t)=I_{\vec{s} \rightarrow \vec{y}-\rho(x)} S(t) u^{\vec{s}-\rho(c)}(x+c)=I_{\vec{s} \rightarrow \vec{y}-\rho(x)} S(t) u^{\vec{s}}(x)=U(x, \vec{y}, t) .
$$

Therefore we can rewrite $U(x, \vec{y}, t)=U(\vec{y}, t)$, the function $U(\vec{y}, t)$ is periodic with values $\vec{y}$ and is expressed as (5). Since $I_{\vec{y} \rightarrow \rho(x)-\vec{s}} U(\vec{y}, t)=u^{\vec{s}}$, we have the following expression:

$$
u^{\vec{s}}(x)=\sum_{m \in \mathbb{Z}^{\ell}} c_{m}(t) \exp \left(i \sum_{j=1}^{\ell} m_{j}\left(r_{j} \cdot\left(x-s_{j}\right)\right)\right) .
$$

We now derive an a priori estimate. First we take existence time $T$ arbitrarily. Since $D^{\ell}$ is compact and the solution is real-valued,

$\sup _{0<t<T}\left\|u^{\vec{s}}\right\|_{L_{x, \vec{s}}^{\infty}\left(\mathbb{R}^{2} \times D^{\ell}\right)}=\sup _{0<t \leq T} \sup _{\vec{s} \in D^{\ell}}\left\|u^{\vec{s}}(t)\right\|_{L^{\infty}\left(\mathbb{R}^{2}\right)}$ is always bounded (see [17, $23,24]$ ). Now we use the mild solution to the equation (1) for each $\vec{s}$ as follows:

$$
u^{\vec{s}}(t)=e^{t \Delta} u_{0}^{\vec{s}}-\int_{0}^{t} e^{(t-\tau) \Delta} \mathbf{P} \nabla \cdot\left(u^{\vec{s}} \otimes u^{\vec{s}}\right)(\tau) d \tau
$$

and

(7)

$$
\left\{\begin{array}{c}
\nabla_{\vec{s}} u^{\vec{s}}(t)=e^{t \Delta} \nabla_{\vec{s}} u_{0}^{\vec{s}}-\int_{0}^{t} e^{(t-\tau) \Delta} \mathbf{P} \nabla \cdot\left(\nabla_{\vec{s}} u^{\vec{s}} \otimes u^{\vec{s}}+u^{\vec{s}} \otimes \nabla_{\vec{s}} u^{\vec{s}}\right)(\tau) d \tau \\
\nabla_{\vec{s}}^{2} u^{\vec{s}}(t)=e^{t \Delta} \nabla_{\vec{s}}^{2} u_{0}^{\vec{s}}-\int_{0}^{t} e^{(t-\tau) \Delta} \mathbf{P} \nabla \cdot\left(\nabla_{\vec{s}}^{2} u^{\vec{s}} \otimes u^{\vec{s}}+2 \nabla_{\vec{s}} u^{\vec{s}} \otimes \nabla_{\vec{s}} u^{\vec{s}}+u^{\vec{s}} \otimes \nabla_{\vec{s}}^{2} u^{\vec{s}}\right)(\tau) d \tau, \\
\vdots
\end{array}\right.
$$

where $\mathbf{P}$ is Helmholtz projection. A direct calculation yields

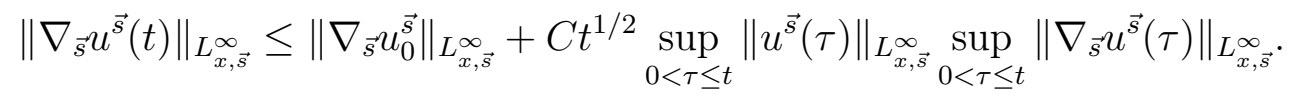

Let us set $t:=1 /\left(2 C \sup _{0<\tau \leq T}\left\|u^{\vec{s}}(\tau)\right\|_{L_{x, \vec{s}}^{\infty}}\right)^{2}$. By absorbing argument, we have

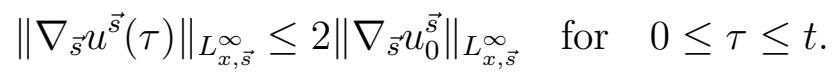

Repeating these arguments for several times, we have

$$
\sup _{0<t<T}\left\|\nabla_{\vec{s}} u^{\vec{s}}(t)\right\|_{L_{x, \vec{s}}^{\infty}} \leq C_{T}^{1}
$$

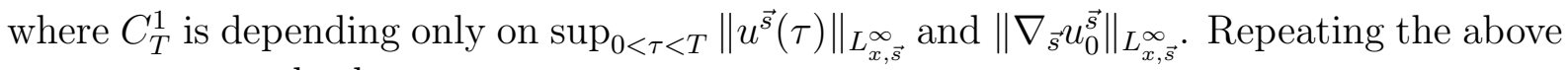
arguments, we also have

$$
\sup _{0<t<T}\left\|\nabla_{\vec{s}}^{\eta} u^{\vec{s}}(t)\right\|_{L_{x, \vec{s}}^{\infty}} \leq C_{T}^{2}
$$

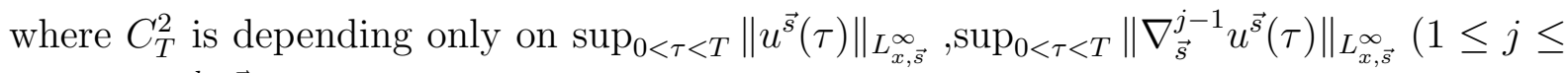
$\eta)$ and $\left\|\nabla_{\vec{s}}^{k} u_{0}^{\vec{s}}\right\|_{L_{x, \vec{s}}^{\infty}}(1 \leq k \leq \eta)$.

Next we estimate $\nabla_{\vec{s}} u^{\vec{s}}$ with the norm $L_{x}^{\infty}\left(L_{\vec{s}}^{2}\right)$. By $(7)$, we easily have

$$
\left\|\nabla_{\vec{s}} u^{\vec{s}}(t)\right\|_{L_{x}^{\infty}\left(L_{\vec{s}}^{2}\right)} \leq\left\|\nabla_{\vec{s}} u_{0}^{\vec{s}}\right\|_{L_{x}^{\infty}\left(L_{\vec{s}}^{2}\right)}+t^{1 / 2} \sup _{0<\tau \leq t}\left\|u^{\vec{s}}(\tau)\right\|_{L_{x, \vec{s}}^{\infty}} \sup _{0<\tau \leq t}\left\|\nabla_{\vec{s}} u^{\vec{s}}(\tau)\right\|_{L_{x}^{\infty}\left(L_{\vec{s}}^{2}\right)} .
$$

By absorbing argument as we calculated before, we have

$$
\left\|\nabla_{\vec{s}} u^{\vec{s}}(t)\right\|_{\substack{L_{x}^{\infty}\left(L_{\vec{s}}^{2}\right) \\ 6}} \leq C_{T}^{3}
$$


where $C_{T}^{3}$ is depending only on $\sup _{0<\tau<T}\left\|u^{\vec{s}}(\tau)\right\|_{L_{x, \vec{s}}^{\infty}}$ and $\left\|u_{0}^{\vec{s}}\right\|_{L_{x}^{\infty}\left(L_{\vec{s}}^{2}\right)}$. Repeating these arguments, we have

$$
\left\|\nabla_{\vec{s}}^{\eta} u^{\vec{s}}(t)\right\|_{L_{x}^{\infty}\left(L_{\vec{s}}^{2}\right)} \leq C_{T}^{4},
$$

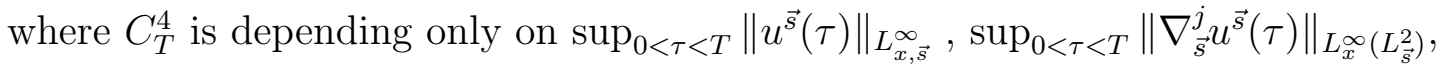

$\sup _{0<\tau<T}\left\|\nabla_{\vec{s}}^{j} u^{\vec{s}}(\tau)\right\|_{L_{x, \vec{s}}^{\infty}}(1 \leq j \leq \eta-1)$ and $\left\|\nabla_{\vec{s}}^{k} u_{0}^{\vec{s}}\right\|_{L_{x}^{\infty}\left(L_{\vec{s}}^{2}\right)}(1 \leq k \leq \eta)$. Combining these estimates, we see that

$$
\sup _{0<\tau \leq T}\|u(\tau)\|_{X^{\eta}} \leq C_{T},
$$

where $C_{T}$ is depending only on $T$ and $\left\|u_{0}\right\|_{X_{\eta}}$. This is the desired estimate.

\section{An EXAMPle of COMPlex unBounded $F M$ SOLUtions}

The $F M$ norm dominates $L^{\infty}$ norm. For $L^{\infty}$ norm it is known that

$$
\|u(t)\|_{\infty} \leq C\left\|u_{0}\right\|_{\infty} \exp \left(C\left\|\omega_{0}\right\|_{\infty} t\right)
$$

for all $t>0$ (see [24]), where $\omega_{0}$ is the initial vorticity. By the regularizing effect the vorticity and velocity at some positive time $t_{0}$ is dominated by $L^{\infty}$ norm of initial data $u_{0}$ and $t_{0}$ which can be chosen based on $\left\|u_{0}\right\|_{\infty}$ (see [9]). Thus we have a bound for $\|u(t)\|_{\infty}$ on $[0, T]$ depending only on $T$ and $\left\|u_{0}\right\|_{\infty}$ provided that $u_{0}$ is real. The existence of a uniform bound in $L^{\infty}$ in $\mathbb{R}^{2} \times(0, \infty)$ is a still open problem. In this section we shall prove that $\|u(t)\|_{F M}$ may diverge to infinity as $t \rightarrow \infty$ at least for some complex initial data. To construct such solutions, we use second approximation of the solution to (1) and extract inflation term from low frequency part. This device is proposed by [6, 26].

Theorem 3.1. Let us set initial value as

$$
u_{0}:=\sum_{j=1}^{\infty} c_{j}\left(a^{0} e^{i k_{\alpha_{j}}^{0} \cdot x}+a^{1} e^{i k_{\alpha_{j}}^{1} \cdot x}\right),
$$

where $\left\{c_{j}\right\}_{j=1}^{\infty},\left\{\alpha_{j}\right\}_{j=1}^{\infty} \subset \mathbb{R}_{+}, \sum_{j=1}^{\infty} c_{j} \leq 1,\left\{\alpha_{j}\right\}$ is linearly independent over $\mathbb{Q}, \alpha_{j} /\left(c_{j}\right)^{2} \rightarrow$ $0(j \rightarrow \infty)$,

$$
a^{0}=\left(\begin{array}{l}
1 \\
0
\end{array}\right), a^{1}=\left(\begin{array}{c}
-1 \\
1
\end{array}\right), k_{\alpha}^{0}=\left(\begin{array}{l}
0 \\
\alpha
\end{array}\right) \quad \text { and } \quad k_{\alpha}^{1}=\left(\begin{array}{l}
\alpha \\
\alpha
\end{array}\right) .
$$

(Note that $\left\|u_{0}\right\|_{F M} \leq 3$ for any $\{\alpha\}_{j}$, and div $u_{0}=0$.) Assume that the solution $u(t)$ to the equation (1) is in $C\left([0, \infty): F M_{0}\right)$. Then we have

$$
\|u(t)\|_{F M} \rightarrow \infty \quad(t \rightarrow \infty) .
$$

Proof of Thorem 3.1. Let us define

$$
u_{\eta}:=\sum_{m+m^{\prime}=\eta, 1 \leq m, m^{\prime} \leq \eta-1} \int_{0}^{t} e^{(t-\tau) \Delta} \mathbf{P} \nabla \cdot\left(u_{m} \otimes u_{m^{\prime}}\right)(\tau) d \tau, \quad \eta=2,3, \cdots
$$

and $u_{1}:=e^{t \Delta} u_{0}$. We easily see that the solution $u$ to the equation (1) is expressed as $u=\sum_{\eta=1}^{\infty} u_{\eta}$. Since the solution is complex valued function, we see that

$$
\operatorname{supp} \hat{u}_{\eta}(\cdot, t) \subset \bigcup_{\left(j_{1}, \cdots, j_{\eta}\right) \in\{1,2, \cdots\}^{\eta}}[0, \infty) \times\left(\left\{\sum_{m=1}^{\eta} \alpha_{j_{m}}\right\}\right) \text { for } t>0
$$


and $\left\{\alpha_{j}\right\}_{j=1}^{\infty}$ is linearly independent over the field of all rational number $\mathbb{Q}$. For example, $\operatorname{supp} \hat{u}_{2}(\cdot, t) \subset[0, \infty) \times\left[\left(\left\{2 \alpha_{1}\right\}\right) \cup\left(\left\{\alpha_{1}+\alpha_{2}\right\}\right) \cup\left(\left\{2 \alpha_{2}\right\}\right) \cup\left(\left\{\alpha_{1}+\alpha_{3}\right\}\right) \cdots\right] \quad$ for $\quad t>0$. Thus we have supp $\hat{u}_{\eta} \cap \operatorname{supp} \hat{u}_{\eta^{\prime}}=\emptyset\left(\eta \neq \eta^{\prime}\right)$. Therefore we can estimate the $F M$ norm of $u$ as follows:

$$
\|u\|_{F M}=\sum_{\eta=1}^{\infty}\left\|u_{\eta}\right\|_{F M} \geq\left\|u_{2}\right\|_{F M} .
$$

We now only calculate the $F M$ norm of $u_{2}$. This is the inflation term controlled by the parameters $\left\{\alpha_{j}\right\}_{j}$. Since $a^{0} \cdot k_{\alpha_{j}}^{0}=a^{1} \cdot k_{\alpha_{j}}^{1}=0$ for $j=1,2, \cdots$, we have

$$
\begin{gathered}
\mathbf{P} \nabla \cdot\left(u_{1} \otimes u_{1}\right)=\mathbf{P} \nabla \cdot \sum_{\sigma_{1}, \sigma_{2} \in\{0,1\}} c_{j} c_{j^{\prime}}\left(a^{\sigma_{1}} \otimes a^{\sigma_{2}}\right) \sum_{j, j^{\prime}=1}^{\infty} e^{i\left(k_{\alpha_{j}}^{\sigma_{1}}+k_{\alpha_{j^{\prime}}}^{\sigma_{2}}\right) \cdot x} e^{-\left(\left|k_{\alpha_{j}}^{\sigma_{1}}\right|^{2}+\left|k_{\alpha_{j^{\prime}}}\right|^{2}\right) t}= \\
\sum_{\sigma_{1} \sigma_{2} \in\{0,1\}} \sum_{j, j^{\prime}=1}^{\infty} c_{j} c_{j^{\prime}} \mathbf{P} e^{i\left(k_{\alpha_{j}}^{\sigma_{1}}+k_{\alpha_{j^{\prime}}}^{\sigma_{2}}\right) \cdot x} a^{\sigma_{2}}\left(a^{\sigma_{1}} \cdot\left(k_{\alpha_{j}}^{\sigma_{1}}+k_{\alpha_{j^{\prime}}}^{\sigma_{2}}\right)\right) e^{-\left(\left|k_{\alpha_{j}}^{\sigma_{1}}\right|^{2}+\mid k_{\alpha_{j^{\prime}}}^{\sigma_{2}}\right) t}= \\
\sum_{j, j^{\prime}=1}^{\infty} c_{j} c_{j^{\prime}} e^{i\left(k_{\alpha_{j}}^{0}+k_{\alpha_{j^{\prime}}}^{1}\right) \cdot x} P_{0,1}\left(\left(a^{1}\left(a^{0} \cdot k_{\alpha_{j}}^{1}\right)+a^{0}\left(a^{1} \cdot k_{\alpha_{j^{\prime}}}^{0}\right)\right) e^{-\left(\left|k_{\alpha_{j}}^{0}\right|^{2}+\mid k_{\alpha_{j^{\prime}}}^{2}\right) t}\right. \\
+\sum_{j, j^{\prime}=1}^{\infty} c_{j} c_{j^{\prime}} e^{i\left(k_{\alpha_{j}}^{0}+k_{\alpha_{j^{\prime}}}^{0}\right) \cdot x} P_{0,0} a^{0}\left(a^{0} \cdot k_{\alpha_{j}}^{1}\right) e^{-\left(\left|k_{\alpha_{j}}^{0}\right|^{2}+\left|k_{\alpha_{j^{\prime}}}^{0}\right|^{2}\right) t} \\
+\sum_{j, j^{\prime}=1}^{\infty} c_{j} c_{j^{\prime}} e^{i\left(k_{\alpha_{j}}^{1}+k_{\alpha_{j^{\prime}}}^{1}\right) \cdot x} P_{1,1} a^{1}\left(a^{1} \cdot k_{\alpha_{j}}^{0}\right) e^{-\left(\left|k_{\alpha_{j}}^{1}\right|^{2}+\mid k_{\alpha_{j^{\prime}}}{ }^{2}\right) t},
\end{gathered}
$$

where

$$
P_{0,1}:=I-\left.\frac{1}{|\xi|}\left(\begin{array}{cc}
\xi_{1}^{2} & \xi_{1} \xi_{2} \\
\xi_{2} \xi_{1} & \xi_{2}^{2}
\end{array}\right)\right|_{\xi=\left(\xi_{1}, \xi_{2}\right)=k_{\alpha_{j}^{0}}+k_{\alpha_{j^{\prime}}^{1}}}=\frac{1}{5}\left(\begin{array}{cc}
4 & -2 \\
-2 & 1
\end{array}\right),
$$

(we define $P_{0,0}$ and $P_{1,1}$ in the same way) $I$ is $2 \times 2$ identity matrix. Here we used $\mathbf{P} e^{i\left(k_{\alpha_{j}}^{0}+k_{\alpha_{j^{\prime}}}^{1}\right) x}=P_{0,1} e^{i\left(k_{\alpha_{j}}^{0}+k_{\alpha_{j^{\prime}}}^{1}\right) x}$. Thus

$$
\begin{gathered}
\left\|u_{2}\right\|_{F M}=\left\|\int_{0}^{t} e^{(t-\tau) \Delta} \mathbf{P} \nabla \cdot\left(u_{1} \otimes u_{1}\right)(\tau) d \tau\right\|_{F M} \geq \\
\sum_{j, j^{\prime}=1}^{\infty}\left|P_{0,1} \alpha_{j} a^{1}+P_{0,1} \alpha_{j^{\prime}} a^{0}\right| c_{j} c_{j^{\prime}} e^{-\left|k_{\alpha_{j}}^{0}+k_{\alpha_{j^{\prime}}}^{1}\right|^{2} t} \int_{0}^{t} e^{2 k_{\alpha_{j}}^{0} \cdot k_{\alpha_{j^{\prime}}}^{1} \tau} d \tau \geq \\
\sum_{j=1}^{\infty}\left|P_{0,1} a^{1}+P_{0,1} a^{0}\right| \alpha_{j}\left(c_{j}\right)^{2} e^{-\left|k_{\alpha_{j}}^{0}+k_{\alpha_{j}}^{1}\right|^{2} t} \int_{0}^{t} e^{2 k_{\alpha_{j}}^{0} \cdot k_{\alpha_{j}}^{1} \tau} d \tau=(\text { RHS }) .
\end{gathered}
$$

Since $e^{2 k_{\alpha_{j}}^{0} \cdot k_{\alpha_{j}}^{1}}=e^{2 \alpha_{j}{ }^{2}} \geq 1,\left|k_{\alpha_{j}}^{0}+k_{\alpha_{j}}^{1}\right|^{2}=5 \alpha_{j}{ }^{2}$ and $\left|P_{0,1} a^{1}+P_{0,1} a^{0}\right|=11 / 5$, we have

$$
(R H S) \geq(11 / 5) \sum_{j=1}^{\infty}\left(c_{j}\right)^{2} \alpha_{j} e^{-5 \alpha_{j}^{2} t} t .
$$


For each $k>0$ the right hand side is estimated from below by $(11 / 5)\left(c_{k}\right)^{2} t \alpha_{k} e^{-5 \alpha_{k}^{2} t}$. Thus

$$
(11 / 5) \sum_{j=1}^{\infty}\left(c_{j}\right)^{2} \alpha_{j} e^{-5 \alpha_{j}{ }^{2} t} t \geq(11 /(25 e))\left(c_{k}\right)^{2} / \alpha_{k} .
$$

Sending $k$ to $\infty$ we observe that $\left(c_{k}\right)^{2} / \alpha_{k}$ tend to the infinity by assumption so we conclude that $\|u(t)\|_{F M} \rightarrow \infty$ as $t \rightarrow \infty$. Thus we have the desired estimate.

\section{Acknowledgments.}

The work of the first author was partly supported by the Grant-in-Aid for Scientific Research, No. 18204011, No. 21224001, the Japan Society for the Promotion of Science (JSPS). The work of the second author was partly supported by AFOSR contract FA955005-1-0047. The work of the third author was partly supported by the Grant-in-Aid for JSPS Fellows and US National Science Foundation during his visit to Arizona State University.

\section{REFERENCES}

[1] A. Babin, A. Mahalov and B. Nicolaenko, Integrability and regularity of the 3D Euler and NavierStokes equations for uniformly rotating fluids. Europ. J. Mech. B/Fluids, 15 (1996), 291-300.

[2] A. Babin, A. Mahalov and B. Nicolaenko, Regularity and integrability of $3 D$ Euler and Navier-Stokes equations for rotating fluids. Asymptot. Anal., 15 (1997), 103-150.

[3] A. Babin, A. Mahalov and B. Nicolaenko, Global regularity of the 3D Rotating Navier-Stokes Equations for resonant domains. Indiana University Mathematics Journal, 48 (1999), 1133-1176.

[4] A. Babin, A. Mahalov and B. Nicolaenko, Global regularity of 3D rotating Navier-Stokes equations for resonant domains. Appl. Math. Lett., 13 (2000), 51-57.

[5] A. Babin, A. Mahalov and B. Nicolaenko, 3D Navier-Stokes and Euler quations with initial data characterized by uniformly large vorticity. Indiana University Mathematics Journal, 50 (2001), 1-35.

[6] J. Bourgain and N. Pavlovic, Ill-posedness of the Navier-Stokes equations in a critical space in 3D. Journal of Functional Analysis, 255 (2008), 2233-2247.

[7] J. Y. Chemin, B. Desjardins, I. Gallagher and E. Grenier, Mathematical geophysics. An introduction to rotating fluids and the Navier-Stokes equations. Oxford Lecture Series in Mathematics and its Applications, 32. The Clarendon Press, Oxford University Press, Oxford, (2006).

[8] Y. Giga, Evolution equations with almost periodic initial data. International Conference for the 25th Anniversary of Viscosity Solutions (eds. Y. Giga, S. Koike, T. Ozawa and N. Yamada), Gakuto International Series, Mathematical Sciences and Applications, Gakkotosho, Tokyo, 30 (2008), 97106.

[9] Y. Giga, K. Inui and S. Matsui, On the Cauchy problem for the Navier-Stokes equations with nondecaying initial data. Advances in fluid dynamics, 27-68, Quad. Mat., 4, Dept. Math., Seconda Univ. Napoli, Caserta, (1999).

[10] Y. Giga, K. Inui, A. Mahalov and S. Matsui, Navier-Stokes equations in a rotating frame in $R^{3}$ with initial data nondecreasing at infinity. Hokkaido Math. J., 35 (2006), 321-364.

[11] Y. Giga, K. Inui, A. Mahalov and S. Matsui, Uniform local solvabilty for the Navier-Stokes equations with the Coriolis force. Methods Appl. Anal., 12 (2005), 381-393.

[12] Y. Giga, K. Inui, A. Mahalov, S. Matsui and J. Saal, Rotating Navier-Stokes equations in $\mathbb{R}_{+}^{3}$ with initial data nondecreasing at infinity: The Ekman boundary layer problem. Arch, Ration. Mech. Anal., 186 (2007), 177-224.

[13] Y. Giga, K. Inui and A. Mahalov and J. Saal, Global solvability of the Navier-Stokes equations in spaces based on sum-closed frequency sets. Adv. Diff. Eq., 12 (2007), 721-736.

[14] Y. Giga, K. Inui and A. Mahalov and J. Saal, Uniform global solvability of the rotationg NavierStokes equations for nondecaying initial data. Indiana University Mathematics Journal, 57 (2008), 2775-2791.

[15] Y. Giga, H. Jo, A. Mahalov and T. Yoneda, On time analyticity of the Navier-Stokes equations in a rotating frame with spatially almost periodic data. Physica D, 237 (2008), 1422-1428. 
[16] Y. Giga, A. Mahalov and B. Nicolaenko, The Cauchy problem for the Navier-Stokes equations with spatially almost periodic initial data. Mathematical Aspects of Nonlinear Dispersive Equations (eds. J. Bourgain et al), 163 (2007), 213-222, Princeton Press.

[17] Y. Giga, S. Matsui and O. Sawada, Global existence of two-dimensional Navier-Stokes flow with nondecaying initial velocity. J. Math. Fluid Mech., 3 (2001), 302-315.

[18] J. Kato, The uniqueness of nondecaying solutions for the Navier-Stokes equations. Arch. Ration. Mech. Anal., 169 (2003), 159-175.

[19] H. Koch and D. Tataru, Well-posedness for the Navier-Stokes equations. Adv. Math., 157 (2001), 22-35.

[20] D. Li and Y. Sinai, Blow ups of complex solutions of the 3D Navier-Stokes system and renormalization group method. J. Eur. Math. Soc., 10 (2008), 267-313.

[21] A. Makhalov and B. Nikolaenko, Global solvability of three-dimensional Navier-Stokes equations with uniformly high initial vorticity. Uspekhi Mat. Nauk 58 (2003), 79-110, translation in Russian Math. Surveys, 58 (2003), 287-318.

[22] E. Nakai and T. Yoneda, Riesz transforms on generalized Hardy spaces and a uniqueness theorem for the Navier-Stokes equations. preprint.

[23] O. Sawada and Y. Taniuchi, On the Boussinesq flow with nondecaying initial data. Funkcial. Ekvac., 47 (2004), 225-250.

[24] O. Sawada and Y. Taniuchi, A remark on $L^{\infty}$ solutions to the 2-D Navier-Stokes equations. J. Math. Fluid Mech., 9 (2007), 533-542.

[25] T. Yoneda, Global solvability of the Navier-Stokes equations in a rotating frame with spatially almost periodic data. preprint.

[26] T. Yoneda Ill-posedness of the 3D-Navier-Stokes equations in a generalized Besov space near $B M O^{-1}$. preprint.

Graduate School of Mathematical Sciences University of Tokyo 3-8-1 Komaba, MeguroKU, TOKYO 153-8914, JAPAN

E-mail address: labgiga@ms.u-tokyo.ac.jp

Department of Mathematics Arizona State University Tempe, AZ 85287-1804, USA

E-mail address: mahalov@asu.edu

Institute for Mathematics And its Applications, University of Minnesota, 114 Lind Hall 207 Church Street S.E. Minneapolis, MN 55455, USA

E-mail address: yoneda@ima.umn.edu 